\title{
Optical Diagnostics of Iron Oxide Nanoparticle Synthesis in Microwave Oxygen Plasma
}

\author{
Miroslav Šnírer, Peter Zelina, and Vít Kudrle
}

\begin{abstract}
Iron oxide nanoparticle synthesis in microwave plasma is studied by optical diagnostics. The arrangement of the plasma reactor results in well-defined reactant mixing, which makes different parts of the reaction zone clearly observable. Besides the reaction zone, digital imaging also reveals the presence of heated nanoparticles. The optical emission spectroscopy is used to determine important plasma parameters.
\end{abstract}

Index Terms-Nanotechnology, optical spectroscopy, plasma CVD, plasma materials processing applications.

$\mathbf{S}$ YNTHESIS of nanomaterials with advanced properties is an important part of nanotechnology and a fast developing field with many promising applications. Magnetic iron and iron-oxide-based nanopowders show great potential in medicine (MRI contrast agent, targeted drugs), chemical catalysis, environmental science (decontamination of toxic wastes), in biochemistry (in vivo and in vitro reactions controlled by magnetic fields) and many other areas of science and technology.

Traditional wet techniques of synthesis of iron-based nanopowders are well established and are able to produce this material at industrial quantities. However, they are rather complicated and use potentially harmful substances during the many steps. In contrast, plasma enhanced chemical vapor deposition (PECVD) is a rather straight-forward process producing nanopowders, which consequently do not need to be dissolved, precipitated, dried, or purified. This makes PECVD an attractive and advantageous method, however, currently only suitable for low-quantity purposes.

The experiment uses a low-pressure microwave plasma reactor to produce iron oxide nanoparticles from $\mathrm{Fe}(\mathrm{CO})_{5}$ precursor vapors. Use of a surface-wave driven discharge permits to spatially separate the excitation and the reaction zones. The waveguide plasma applicator-surfaguide [1], is used to couple the microwave power into the plasma. The discharge is sustained in oxygen atmosphere by $2.45 \mathrm{GHz}$ microwaves using a magnetron generator with adjustable output power in the range of $150-1500 \mathrm{~W}$. The plasma is contained in fused

Manuscript received November 1, 2013; revised March 4, 2014; accepted April 24, 2014. Date of publication May 20, 2014; date of current version October 21, 2014. This work was supported by the Project Research and Development Center for Low-Cost Plasma and Nanotechnology Surface Modifications under Grant CZ.1.05/2.1.00/03.0086.

The authors are with the Department of Physical Electronics, Masaryk University, Brno 611 37, Czech Republic (e-mail: snirer@mail.muni.cz; zelina@mail.muni.cz; kudrle@sci.muni.cz).

Color versions of one or more of the figures in this paper are available online at http://ieeexplore.ieee.org.

Digital Object Identifier 10.1109/TPS.2014.2321995 silica tube ( $1 \mathrm{~m}$ long, $45 \mathrm{~mm}$ diameter). The synthesis is operated in the flow regime, flow of oxygen being set by a mass flow controller. Vacuum is sustained by an oil rotary vane pump. Typical operating pressure during the synthesis is $\sim 1-2 \mathrm{kPa}$ depending on the oxygen flow.

Saturated vapor pressure of iron pentacarbonyl at room temperature is higher than the typical operating pressure of the apparatus, therefore, vapors flow from an evaporator into the reaction zone freely. Their flow is only limited by the vacuum conductance of the tubing, the surface area of the evaporator, and a throttling valve. Vapors are injected into the plasma flow by a pyrex tube $(8 \mathrm{~mm}$ outer diameter, $5.2 \mathrm{~mm}$ inner diameter) reaching as high as $22 \mathrm{~cm}$ below the surfaguide. After entering the plasma, vapors are dissociated mainly into iron and carbonyl. The iron atoms oxidize and agglomerate immediately due to the presence of oxygen plasma, forming iron oxide nanoparticles, which are stable under air atmosphere [2]. The nanopowder is collected on finemesh filter at the output of the plasma reactor. Typical size of nanoparticles is in $10-50 \mathrm{~nm}$ range [2].

High-resolution digital images are taken using Canon EOS 50D camera with Canon EF-S 17-85 mm f/4-5.6 IS USM lens. Optical emission spectroscopy is carried out using Jobin Yvon TRIAX 550 spectrometer with CCD detector and an optic fiber placed perpendicularly to the discharge vertical axis. Spectral sensitivity of the instrument was calibrated using a tungsten halogen lamp.

Counter-flowing iron pentacarbonyl does not turbulently mix with oxygen. Instead it forms a glowing mushroomlike shape [Fig. 1(a)]. We can distinguish two parts of the mushroom - a cap and a stem. The mushroom becomes smaller as the operating pressure reaches the vapor saturation pressure of $\mathrm{Fe}(\mathrm{CO})_{5}$.

The long cylindrical stem forms as a result of oxidizing precursor vapors. Undissociated iron pentacarbonyl flows through the center, oxidizing mainly at its edges. It has yellow-orange color. The length and shape of the stem depend on the experimental conditions. Its length increases with decreasing oxygen flow while the edges become more diffused due to lower total pressure [Fig. 1(b)]. The length of the stem also depends on the $\mathrm{Fe}(\mathrm{CO})_{5}$ flow rate.

The cap formed on the top of the stem is predominantly orange and much brighter that the stem. This suggests excessive heating in this region due to the exothermic iron oxidation. The baseline of the spectrum (i.e. without the spectral lines of $\mathrm{Fe}$ and $\mathrm{O}$ ) was fitted with the blackbody spectrum giving the temperature of $1850 \mathrm{~K}$. Dark current was not subtracted 


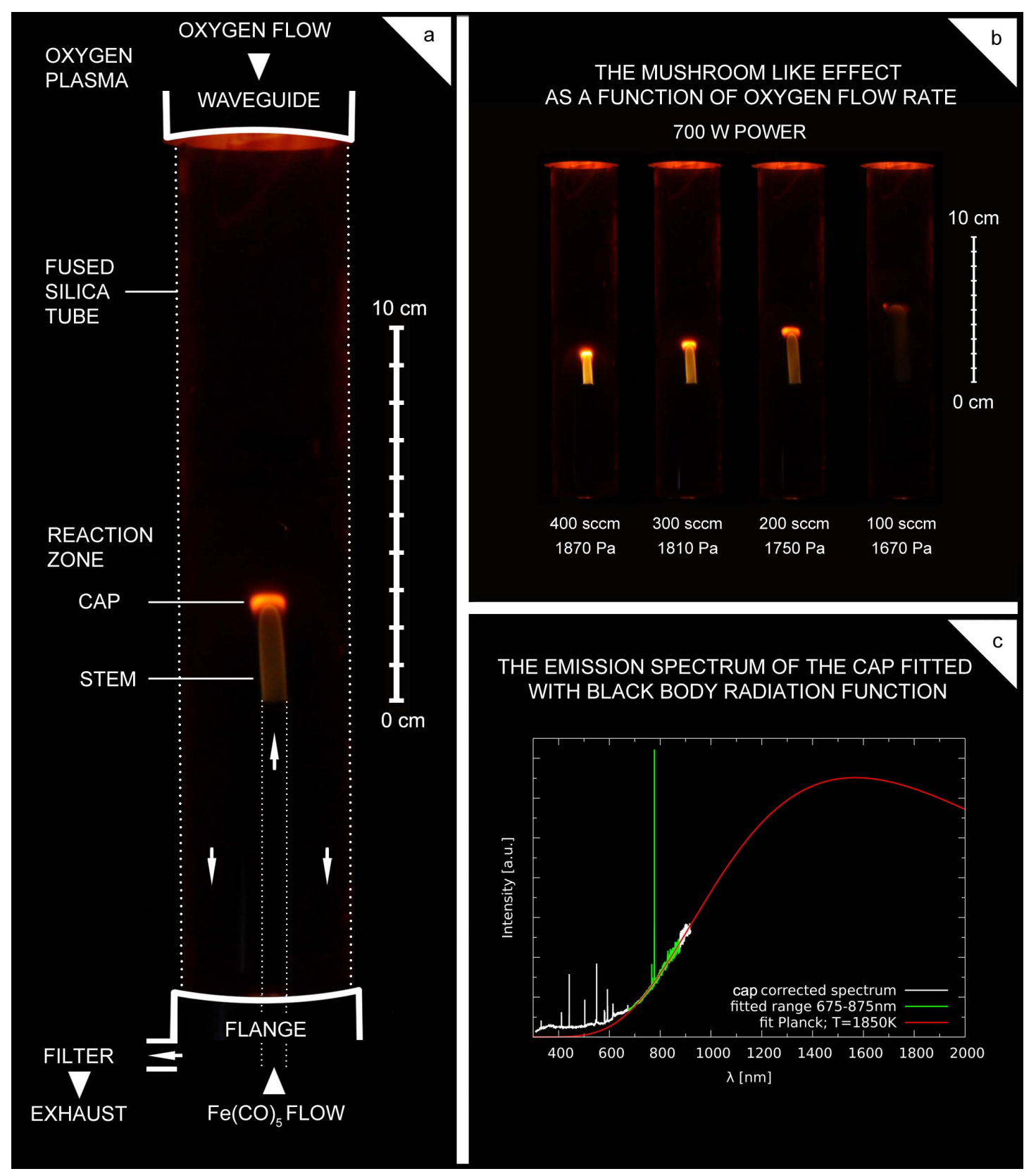

Fig. 1. Oxygen plasma with $\mathrm{Fe}(\mathrm{CO})_{5}$ admixture during nanoparticle synthesis. (a) Mushroom-like effect formed by counter flowing iron pentacarbonyl has two components-a cap and a stem. (b) Its shape as a function of oxygen flow (700 W power, F/5.6, 1/5s, ISO 800, $f=47 \mathrm{~mm}$ ). (c) Emission spectrum of the cap corrected for spectral sensitivity, fitted with the black body radiation function (power $500 \mathrm{~W}, \mathrm{O}_{2}$ flow $200 \mathrm{sccm}$ ).

from the detector signal and hence, the region below $675 \mathrm{~nm}$ differs from the Planck spectrum.

Optical diagnostics is able to visualize phenomena occurring during the synthesis of nanoparticles and provide valuable information, hence, improving our understanding of the processes involved. It can also serve as a basis for optimization and future enhancements, e.g. in situ functionalization.

\section{REFERENCES}

[1] M. Moisan and Z. Zakrzewski, "Plasma sources based on the propagation of electromagnetic surface waves," J. Phys. D, Appl. Phys., vol. 24, no. 7, p. 1025,1991 .

[2] P. Zelina et al., "Versatile low-pressure plasma-enhanced process for synthesis of iron and iron-based magnetic nanopowders," World J. Eng., vol. 9, no. 2, pp. 161-166, 2012. 